\title{
The role of MRI in the diagnosis and treatment of gastric cancer
}

\author{
Yingjing Zhang \\ Jianchun Yu
}

From the Department of General Surgery (J.Y. $\square$ yu-jch@163.com), Peking Union Medical College Hospital, Chinese Academy of Medical Sciences and Peking Union Medical College, Beijing, China.

Received 17 July 2019; revision requested 14 August 2019; last revision received 09 September 2019; accepted 30 September 2019.

Published online 13 March 2020.

DOI 10.5152/dir.2019.19375

\section{ABSTRACT}

Gastric cancer is the fifth most common malignancy and the third leading cause of cancer-related death worldwide, with more than $40 \%$ of new cases occurring in China. With the advancement of treatment methods, the application of adjuvant therapy and targeted drugs, the prognosis of patients with gastric cancer has been significantly improved. In recent years, more and more studies have reported that magnetic resonance imaging (MRI) showed great value in clinical application among patients with gastric cancer, including preoperative staging, treatment response evaluation, predicting prognosis and histopathological features, treatment guidance, and molecular imaging. The remarkable research progress of MRI in gastric cancer will provide new evaluation and treatment approaches for clinical diagnosis and treatment. This article aims to review the current status of the application and research progress of MRI in patients with gastric cancer.

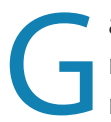
astric cancer is one of the major malignancies in the world, with more than 1000000 new diagnoses and 783000 estimated deaths in 2018, making it the fifth most commonly diagnosed cancer and the third leading cause of cancer-related death worldwide (1). There were 679000 new cases and 498000 deaths occurring in China, ranking in second place behind lung cancer in terms of morbidity and mortality (2). In recent years, the incidence, diagnostic criteria, and treatment strategy of gastric cancer have changed a lot. With the advancement of treatment methods, the application of neoadjuvant therapy and targeted drugs, the prognosis of patients with gastric cancer has been significantly improved. According to CONCORD-3 (3), from 2010 to 2014, the age-standardized five-year net survival of gastric cancer has reached $35.9 \%$ in China, making great progress compared with 2005-2009 (33.2\%) and 2000-2004 (30.2\%).

Surgery remains the main curative treatment approach for gastric cancer, while the surgical options and the extent of lymph node dissection depend on the tumor staging (4). With the development of endoscopic ultrasonography (EUS), computed tomography (CT), magnetic resonance imaging (MRI), positron emission tomography (PET), PET/CT, PET/MRI and laparoscopic exploration, the accuracy of preoperative staging in patients with gastric cancer has been significantly improved. Accurate preoperative staging is the basis for comprehensive treatment of gastric cancer, which plays an essential role in selecting optimal therapeutic approaches and predicting prognosis. In times of personalized therapy and multidisciplinary team approach for treatment, clinicians pay more and more attention to the value of medical imaging for the diagnosis and treatment in gastric cancer.

In recent years, MRI has been increasingly applied in the clinical diagnosis and treatment of gastric cancer, which has high soft-tissue contrast and imaging capacity of multiangle, multidirection, and multiparameter. With the development of fast sequence, MRI provides high-resolution images and key information for lesion localization and qualitative diagnosis without radiation hazards. To reduce the motion artifacts due to gastric peristalsis, air and patient breathing, a series of methods were applied involving filling the stomach cavity by drinking water and oral contrast medium (800-1000 mL), administration of scopolamine, the application of breath-hold MRI techniques and so on. The basic MRI protocols for gas- 
tric cancer include the use of T1-weighted and T2-weighted sequences based on fast spin-echo and spoil gradient (5). Moreover, functional MRI, such as diffusion-weighted imaging (DWI) and intravoxel incoherent motion MRI (IVIM), has gained much attention in the application of tumor imaging, adding a wealth of information to anatomical (T1- and T2-weighted) and dynamic contrast-enhanced (DCE) MRI and providing notable contrast between tumor and normal structures $(6,7)$. The DWI protocols use at least two $b$ values $\left(b=0 \mathrm{~s} / \mathrm{mm}^{2}\right.$ and other $b$ values from 0 to $1000 \mathrm{~s} / \mathrm{mm}^{2}$ ), and the high $b$ value images show greater signal attenuation to detect the pathological lesions and have a reasonable signal-to-noise ratio. The apparent diffusion coefficient (ADC) is calculated and generated as a parametric map. The region with higher signal intensity on DWI exhibits as a low-signal intensity on the ADC map. The ADC value can be obtained through drawing the region of interest (ROI) on these maps from the whole tumor lesion (8). The T2-weighted MRI is performed followed by a DWI and a T1-weighted DCE MRI during intravenous injection of $0.1 \mathrm{mmol} /$ kg Gd-DTPA. Some newly developed techniques such as free-breathing, radial, stackof-stars three-dimensional (3D) gradient echo (GRE) sequence have higher signal-tonoise ratios, higher contrast-to-noise ratios and fewer artifacts, which possess potential value in DCE MRI of gastric cancer (9).

The application of MRI has made great advancement in preoperative staging and perioperative evaluation of gastric cancer, including TNM staging, histopathological features, and perioperative therapeutic efficacy. Therefore, clinicians could determine the most appropriate surgical and treatment planning based on results from laboratory, imaging, and pathology. MRI has been widely demonstrated that it can

\section{Main points}

- In this review, we aimed to consolidate the recent research results on MRI application in gastric cancer.

- MRI showed remarkable performance in preoperative staging, treatment response evaluation, predicting prognosis and histopathological features, treatment guidance, and molecular imaging of gastric cancer.

- Here, we offer a comprehensive and critical evaluation for this noninvasive and nonradiative imaging modality. serve as a useful tool in the local staging and assessment of treatment response for rectal cancer (10). Whereas, the application value of MRI in patients with gastric cancer has not yet reached consensus. In this review, we will introduce the clinical application and progression of MRI related to preoperative staging, perioperative therapeutic efficacy, prognosis, histopathological features, treatment guidance, and molecular imaging among patients with gastric cancer.

\section{MRI in preoperative staging}

\section{Application of EUS and CT}

The eighth edition of the AJCC Cancer Staging Manual has separated the conventional TNM classification system as the clinical stage (cStage), the pathological stage (pStage) and the post-neoadjuvant therapy stage (ypStage). These additional two new stage grouping classifications will provide essential prognostic information and critical guidance for clinicians to make decisions (11). EUS and CT are the preferred imaging modalities used to determine clinical TNM staging, while MRI and PET-CT serve as the imaging tool to further refine staging.

Currently, EUS and CT are widely used in assessing tumor depth invasion (T) and regional lymph node invasion (N). EUS combined with endoscopy allows a unique opportunity to visualize the tumor and acquire a biopsy sample (12). In addition, EUS can detect all the wall layers of stomach and assess the tumor infiltration depth efficiently (13), with the diagnostic accuracy ranging from $65 \%$ to $92.1 \%$ for $T$ stage and $66 \%$ to $90 \%$ for $\mathrm{N}$ stage $(12,14,15)$. Multi-row detector computed tomography (MDCT) scanner has allowed faster scanning, multiplanar reconstructions, 3D image reconstruction, virtual endoscopy, and contrast-enhanced examination, which markedly improve the detection rate of gastric cancer (16-18). The overall diagnostic accuracy of MDCT was reported to range from $77.1 \%$ to $88.9 \%$ for $\mathrm{T}$ staging, and from $46 \%$ to $92 \%$ for $\mathrm{N}$ staging $(12,19-21)$. Furthermore, the CT of thorax and abdominopelvic region is the principal modality to evaluate $M$ stage, with the sensitivity and specificity ranging from $14 \%$ to $59.1 \%$ and $93.3 \%$ to $99.8 \%$ (22). Of note, EUS is invasive and highly operator-dependent, which is limited in detecting invasion outside the serosa and distant metastasis. Since MDCT involves ionizing radiation and contrast agents, it is unavailable for some special patients, such as pregnant women or patients with renal dysfunction.

\section{MRI to assess TNM classification}

MRI has the significant merit of superior soft tissue contrast and multiple imaging sequences without radiation. More and more attention has been focused on its application in tumor detection and perioperative assessment among patients with gastric cancer. A number of previous studies have shown that MRI plays a remarkable role in preoperative staging, with an accuracy ranging from $71.4 \%$ to $88 \%$ for $T$ staging and $52 \%$ to $55 \%$ for nodal involvement $(12$, 23-25). A recent meta-analysis including 439 patients with gastric cancer reported that pooled sensitivity and specificity of MRI to diagnose T stage (T3-4 vs. T1-2) were $93 \%$ and $91 \%$, and $\mathrm{N}$ stage (N0 vs. $\mathrm{N}+$ ), $86 \%$ and $67 \%$, respectively.

Nowadays, DCE has been a routine technique in the MRI. Functional MRI, mainly DWI, has been increasingly used in assessing TNM classification in gastric cancer, especially for patients who cannot bear enhanced CT scanning. A study enrolled 51 patients with gastric cancer reported that the diagnostic accuracy of DWI in T staging, lymph node staging and distant metastasis was comparable to MDCT, and DWI performed better in detecting lymph nodes metastasis (26). Liu et al. (27) have highlighted the application of DWI in assessing $T$ stage for gastric cancer patients. They reported that the overall accuracy of T2-weighted+DCE+DWI in $\mathrm{T}$ staging was significantly higher compared to T2-weighted+CE and T2-weighted+DWI. Caivano et al. (28) and Jang et al. (29) have shown that the combination of DWI and conventional MRI (T1- and T2-weighted ) can make an increase of $7 \%, 5 \%$ and $13 \%, 6 \%$ in the $T$ and $\mathrm{N}$ staging accuracy, respectively. In another study, with the addition of DWI to conventional MRI, an increase of $36.7 \%$ in the sensitivity for differentiating $\mathrm{N}$ stage was reported (30) (Fig. 1, Fig. 2).

Currently, the shape and size of lymph node is used as the diagnostic criteria for nodal metastasis, which is not a reliable indicator. Controversy persists in the criteria of the diameter of the lymph nodes for metastasis due to the high frequency of reactive or inflammatory enlargement of benign lymph nodes and micrometastasis of small-sized lymph nodes in gastric cancer patients $(31,32)$. Indeed, the application of DWI and lymphotropic contrast agent have 

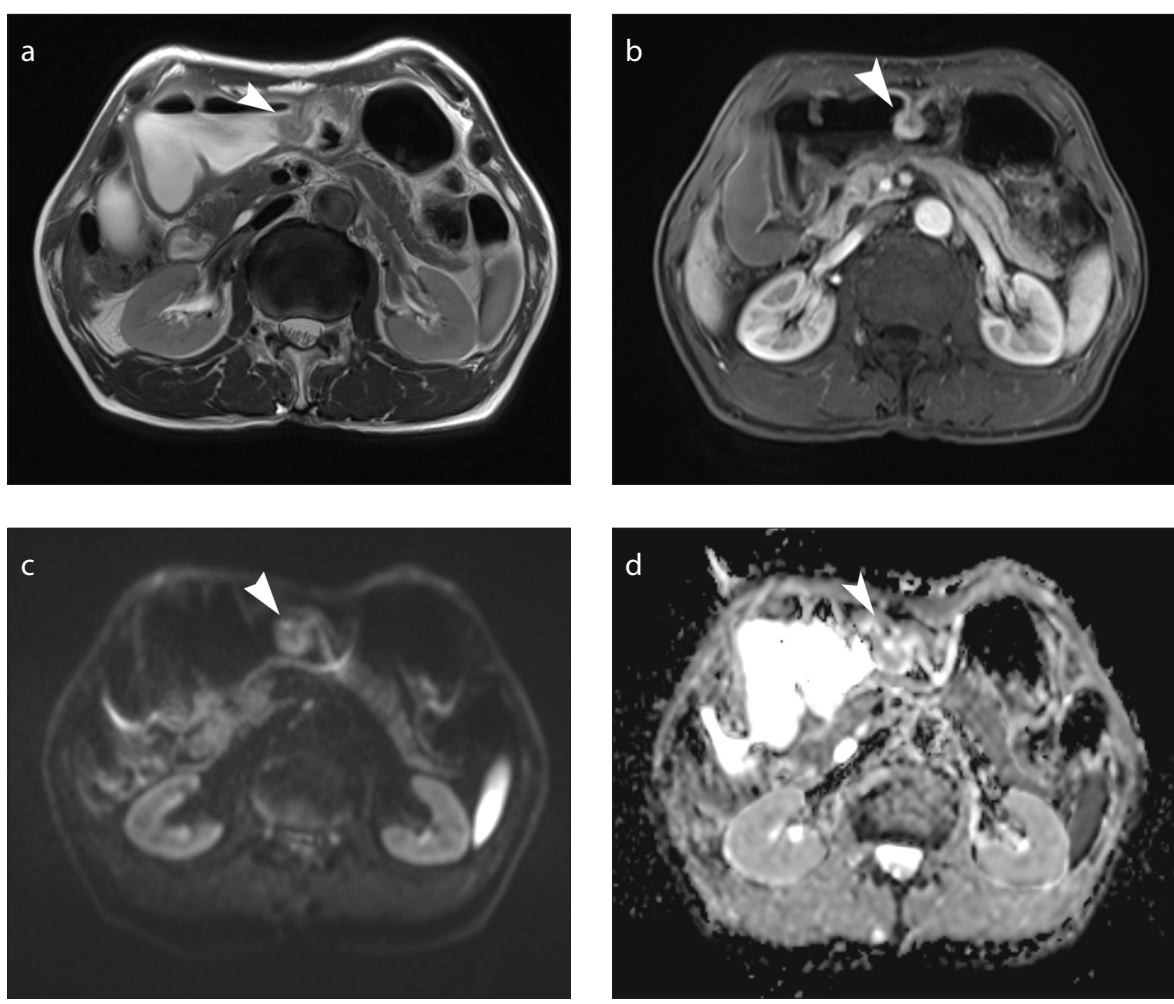

Figure 1. a-d. Early gastric cancer (T1 lesion). Axial T2-weighted MRI (a), axial dynamic contrast-enhanced (DCE) MRI (b), axial diffusion-weighted imaging (DWI, $b=800 \mathrm{~s} / \mathrm{mm}^{2}$ ) (c), and apparent diffusion coefficient (ADC) map (d) show a nodular mass (arrowhead) in the body of the stomach.
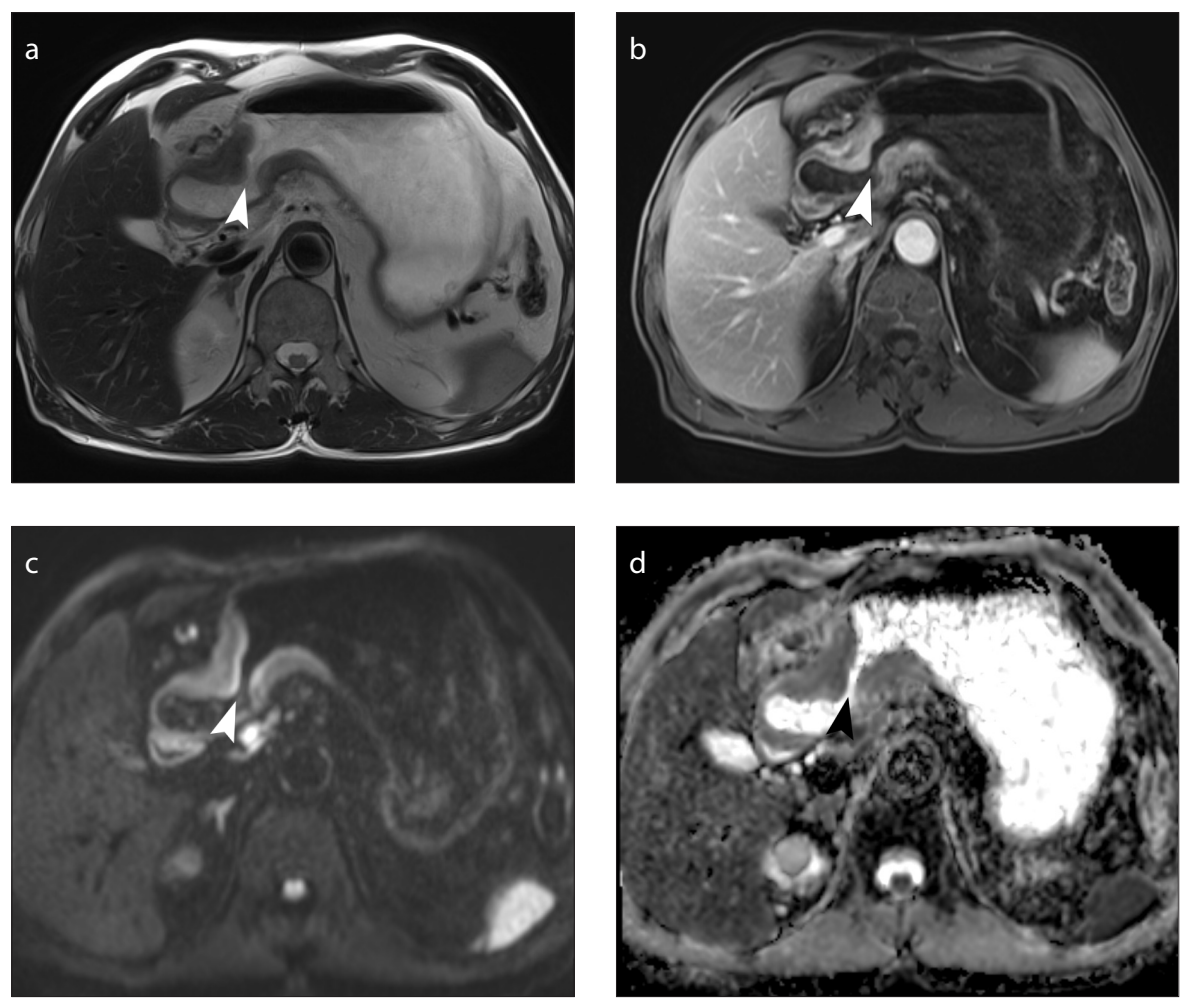

Figure 2. a-d. Advanced gastric cancer (T4 lesion). Axial T2-weighted MRI (a), axial DCE MRI (b), axial DWI $\left(b=800 \mathrm{~s} / \mathrm{mm}^{2}\right)$ (c) and ADC map (d) show gastric wall thickening (arrowhead) in the antrum of the stomach. greatly improved the diagnostic accuracy of nodal stages. A study supported that ferumoxtran-10-enhanced MRI is efficient for the detection of metastatic lymph nodes. The sensitivity, specificity, and overall predictive accuracy can reach $100 \%, 92.6 \%$, and $94.8 \%$, respectively (33).

The ADC value obtained from DWI has been used for quantitative analysis as an imaging biomarker, which offers extensive information about tumor tissue for distinguishing malignant lesions from benign ones and detecting metastatic lymph nodes $(6,7)$. Giganti et al. (34) reported that not only was the ADC values significantly lower in the local invasive tissue and metastatic lymph nodes, but the lower ADC values were associated with the higher TNM classification as well. A similar result can also be observed in another study conducted by Liu et al. (35). Some previous studies have reported the value of DWI combined with ADC measurement to distinguish metastatic lymph nodes from benign ones in gastric cancer. With a cutoff value of $1.39 \times 10^{-3} \mathrm{~mm}^{2} / \mathrm{s}$, Cheng et al. (36) found the $A D C$ values could differentiate metastatic nodes from benign ones with a sensitivity of $85.7 \%$ and specificity of $79.4 \%$. DWI showed higher diagnostic accuracy than the morphological measurements based on short-axis, border irregularity, and enhanced patterns. Both Shinya et al. (37) and Zhong et al. (38) reported the promising role of DWI in detecting lymph node metastasis. In contrast, Hasbahceci et al. (39) suggested that DWI should not be selected as the preferred imaging approach for lymph node staging in gastric cancer, as the overall accuracy in detecting metastatic lymph nodes were $69.56 \%, 65.21 \%$, and $52.17 \%$ for group II, IIb, and Ila lymph nodes. The overall accuracy of $\mathrm{N}$ staging was only $13 \%$, but the researchers acknowledged some limitations to their study, such as the small number of patients and lack of motion correction maneuvers. Therefore, combining DWI with CT or conventional morphological MRI could markedly improve the diagnostic performance for preoperative $\mathrm{N}$ staging in gastric cancer (Fig. 3).

Some previous studies have analyzed that DWI showed similar high diagnostic accuracy in detecting distant metastases from gastrointestinal tumor compared with FDG$\mathrm{PET} / \mathrm{CT}$ and MDCT, especially peritoneal implants and small hepatic metastatic lesions $(30,40)$ (Fig.4). Lee et al. (41) analyzed pa- 

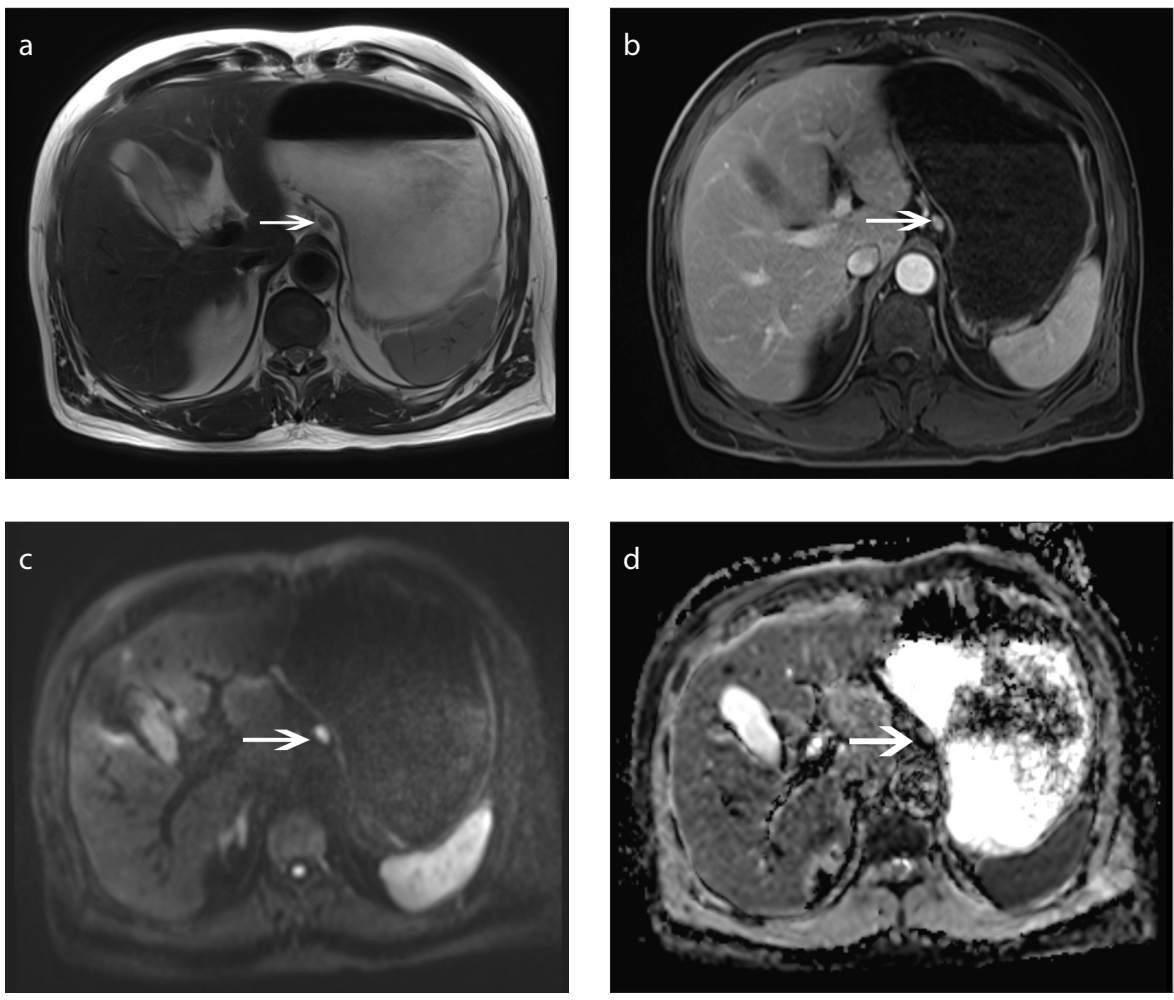

Figure 3. a-d. Metastatic lymph node. Axial T2-weighted MRI (a), axial DCE MRI (b), axial DWI ( $b=800$ $\mathrm{s} / \mathrm{mm}^{2}$ ) (c) and ADC map (d) show a metastatic lymph node (arrow) near the lesser curvature of the stomach.

tients with gastric cancer who underwent ${ }^{18} \mathrm{~F}-\mathrm{FDG}$ PET/MRI and MDCT, and demonstrated that compared with MDCT, PET/MRI performed the comparable diagnostic capability in T and $\mathrm{N}$ staging and even showed higher accuracy in preoperative $M$ staging (92.9\% vs. $73.8 \%-81.0 \%)$. Recently a study reported that ${ }^{18} \mathrm{~F}-\mathrm{FDG}$ PET/MRI performed more precisely in pretreatment TNM staging of gastric cancer compared with PET/CT, especially for N staging (T: $76.9 \%$ vs. $57.7 \%$; $\mathrm{N}: 53.9 \%$ vs. $34.0 \%$ ) (42). This promising modality exploits the superior soft-tissue contrast and functional imaging of MRI, which would provide high-resolution anatomic information for staging and evaluation.

\section{MRI in evaluating treatment response}

Surgery is the fundamental curative option for gastric cancer patients. The surgical procedure of regional gastric cancer (radical gastrectomy and D2 lymphadenectomy) is based on solid clinical evidence. However, most patients with gastric cancer are not diagnosed until advanced stage, with poor prognosis after surgical resection. In recent years, patients with locally advanced gastric cancer gradually underwent perioperative comprehensive treatment $(43,44)$. Imaging approaches are used to assess treatment response of tumors by clinicians. The volume reduction of tumor lesions is difficult to be observed in the early period of therapy, which cannot serve as a good indicator to monitor treatment response.

Compared with $\mathrm{CT}$ or conventional morphological MRI, DWI is able to detect subtle or early tumor changes $(45,46)$. Tumor regression grade (TRG) has been widely used as an indicator of response to treatment for tumors, as a five-level pathologic score system (47). A study conducted by De Cobelli et al. (48) has shown significant negative correlation between $\triangle A D C$ and TRG grades among patients with advanced gastro-esophageal cancer. Compared with non-responders (TRG 4-5), the pre-neoadjuvant therapy ADC values in responders (TRG 1-3) were lower and significantly increased after treatment. However, there was no evidence that $\Delta \mathrm{V}$ was related to TRG in two groups, confirming that it is not appropriate to evaluate early treatment response through tumor volume reduction only (48). Similar subsequent results showed that both post-neoadjuvant therapy $A D C$ and $\triangle A D C$ values can serve as useful indicators to reflect therapy effectiveness (49).

DWI can serve as a more sensitive tool to monitor metastatic lymph nodes responding to neoadjuvant chemotherapy in an early stage, compared with CT or conventional morphological MRI. Zhong et al. (50) reported that the mean ADC values of metastatic lymph nodes on different time points following standard chemotherapy were all higher than those before chemotherapy in complete response group, partial response group, and stable disease group. Of note, the changes of mean ADC values of metastatic lymph nodes can be observed apparently as early as the third day of chemotherapy.

A preliminary study has demonstrated the feasibility of ${ }^{18} \mathrm{~F}-\mathrm{FDG}$ PET/MRI in patients with unresectable advanced gastric cancers. However, both SUV max $_{\text {ax }}$ and ADC values were not significantly different between responders and nonresponders after chemotherapy (51). Furthermore, some animal studies have presented the potential value of IVIM-DWI in evaluating chemotherapy response in gastric cancer. Of note, fast diffusion parameters derived from bi-exponential IVIM model performed more sensitively than ADC value $(52,53)$. The research progress in this field will promote more accurate assessment of therapy effectiveness and individualized treatment strategy.

\section{MRI in predicting histopathological features}

In recent years, some studies have reported that MRI can predict histopathological features of gastric cancer in a noninvasive way. Liu et al. (54) found that with decrease of histological differentiation from well to poor, the ADC values of tumor decrease gradually. In terms of the Lauren classification, intestinal type tumors exhibited significantly higher ADC values over the diffuse type, which was related to more distorted and narrower intercellular spaces in the diffuse type. The same team recently reported that whole-volume ADC-based entropy parameters could provide valuable information about the gastric cancer's aggressiveness in the pretreatment (55). Another study demonstrated the correlation between entire tumor volume-based histogram analysis of the ADC maps and histological differentiation degree of gastric cancer (56). In addition, parameters derived from DCE MRI, such as volume transfer coef- 

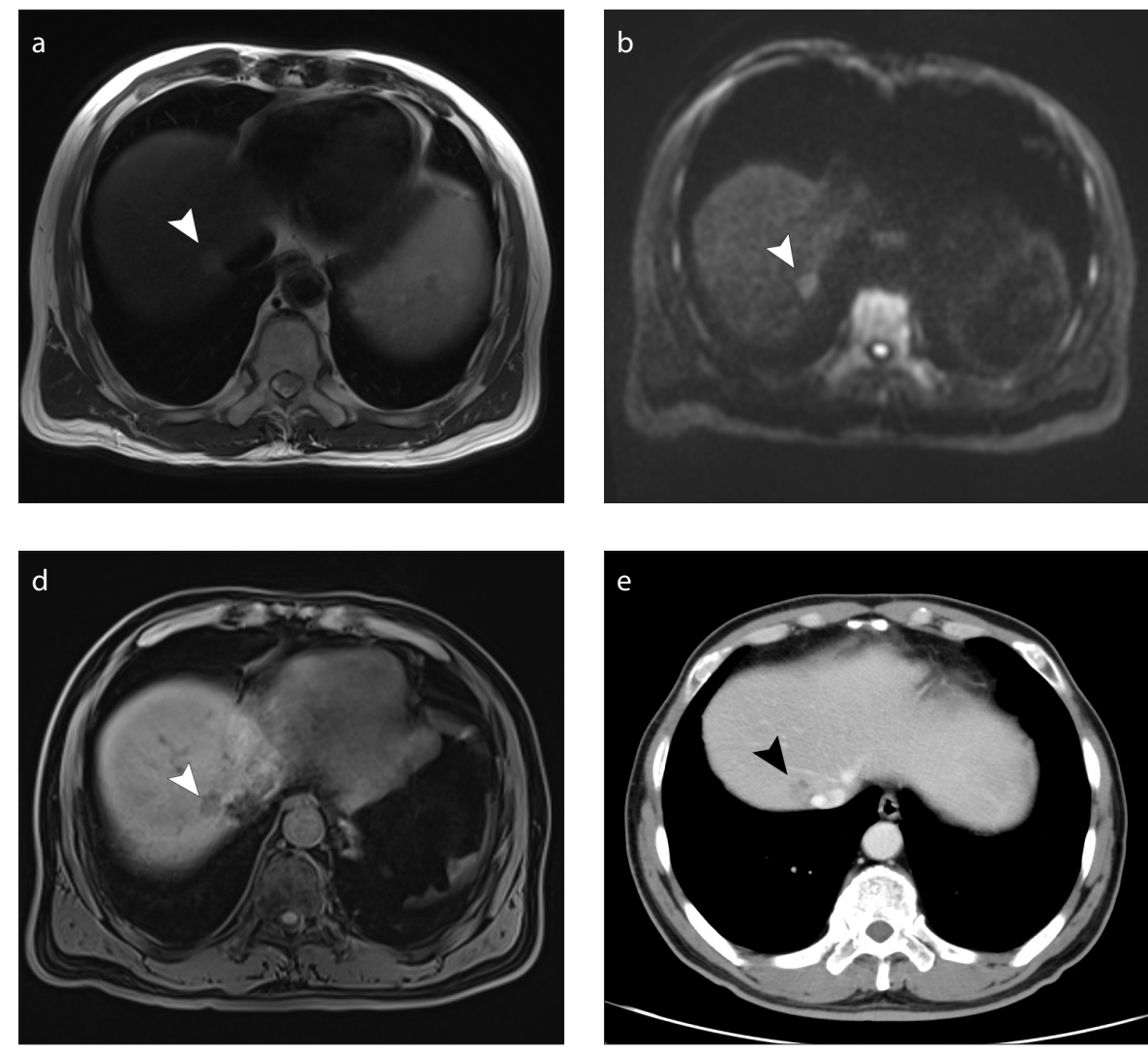

ficient $\left(K^{\text {trans }}\right)$ and extracellular extravascular volume fraction $\left(V_{\mathrm{e}}\right)$, could also predict histopathological classification and evaluate tumor new vessels in gastric cancer (57).

Nowadays, biopsy or surgical specimens from patients are carried out with immunohistochemistry (IHC) or fluorescence in situ hybridization (FISH) to obtain the information of human epidermal growth factor receptor-2 (HER2) scores and status. Ji et al. (58) reported that significantly higher ADC and pure diffusion coefficient (D) values measured from IVIM-MRI were found in HER2 positive tumors than negative ones. In further studies, they identified that the ADC and $D$ values of tumor decreased gradually with decreasing histological differentiation. Moreover, intestinal type and mixed type tumors exhibited higher ADC and D values than the diffuse type (59). These IVIM-MRI based parameters could provide a new preoperative assessment method to obtain histopathological information of stomach tumor.

\section{MRI and molecular imaging}

A new whole-body multimodality imaging system combining 3D fluorescence imaging with MRI not only helped detect peritoneal micrometastasis from gastric cancer with high sensitivity, specificity, and safety at an early stage in mice, but it could also visualize accurate anatomical location of metastasis via fusing optical and MRI images (60). Another study showed that specific MRI/optical dual modality molecular probe could selectively gather in gastric tumor and specifically target new angiogenesis with high stability (61). Recently, a Chinese study found that micron-sized superparamagnetic iron oxide particle (MPIO) based cellular MRI tracking could be used to monitor gastric cancer cells and detect regional lymph node micrometastases in mice (62). In addition, a new HER2-targeted MRI contrast agent, iron oxide nanoparticles conjugated with anti-HER2 single-chain antibody (scFv-IONPs), enabled HER2-specific tumor MRI (63). Some gastric cancer-targeting and MRI-visible nanocarriers for siRNA delivery, with molecular image tracing capacity, have been applied in the area of cancer diagnosis and therapy effectively $(64,65)$.

\section{MRI and radiotherapy}

MRI could directly show tumor and surrounding structures at risk, which is a suitable and multifaceted imaging modality for radiotherapy. MRI-guided radiotherapy

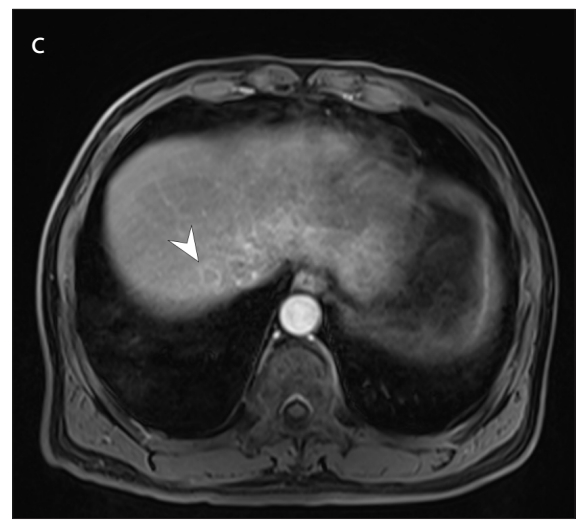

Figure 4. a-e. Hepatic metastasis lesion (arrowhead) near the portal vein of right lobe of the liver shows long T2 signals on axial T2weighted MRI (a), high signal intensity on axial DWI $\left(b=800 \mathrm{~s} / \mathrm{mm}^{2}\right)(\mathbf{b})$, ring-enhancement on arterial phase axial DCE MRI (c), a weak enhancement on portal phase axial DCE MRI (d) and enhancement on DCE multi-row detector CT (e).

provides real-time imaging and superior soft-tissue contrast to describe and track morphological and anatomical motion, which enables daily adaptation of treatment strategy based on interfraction and intrafraction due to geometric and anatomical changes $(66,67)$. A recent case report described a recurrent gastric cancer patient with multiple comorbidities who underwent MRI-guided adaptive radiotherapy (68). This new technology could offer more accurate definition of target and risk organ and individualized daily adaptation of radiotherapy planning. Another case report suggested that DWI was superior in evaluating the effect of hemostatic radiotherapy for unresectable advanced gastric cancer (69). Hence, imaging biomarkers derived from this system may predict the treatment response and accordingly modify the strategy in a timely manner.

\section{MRI in predicting prognosis}

The ADC values have been demonstrated to be associated with survival outcomes in patients with various types of cancer (70, 71). Referring to final histological results, Giganti et al. (34) have shown that an ADC value of $1.36 \times 10^{-3} \mathrm{~mm}^{2} / \mathrm{s}$ or lower was as- 
sociated with a negative prognosis. A later prospective study has shown that ADC values of $1.5 \times 10^{-3} \mathrm{~mm}^{2} / \mathrm{s}$ or lower could indicate a poor outcome in both surgery alone and surgery with neoadjuvant chemotherapy groups (72).

\section{Conclusion}

The current role of MRI in gastric cancer is as an imaging tool to further refine preoperative staging and treatment response evaluation, and provide more valuable information for diagnosis and treatment, particularly for patients who cannot receive iodine contrast agents and those with peritoneal implants and small hepatic metastatic lesions. The application of MRI has not yet achieved wide consensus in the diagnosis and treatment of gastric cancer, while EUS and CT continue to be the primary imaging modalities in clinical decision making. There is no standardization and criteria to define tumor depth invasion, regional lymph node and distant metastases on MRI. A worldwide consensus on criteria to precisely assess treatment response is urgently needed. Moreover, there is no uniform protocol for MRI scan and calculation method of ADC values in gastric cancer. Of note, the $A D C$ values and ranges are inconsistent for pathological lesions and metastatic lymph nodes. The cystic and necrotic fraction and the cellular solid tumor areas show different degrees of signal attenuation from water molecules due to heterogeneity of the stomach tumor. Further research is needed to explore the application range and significance of MRI in gastric cancer.

In conclusion, this review highlights the remarkable value of $\mathrm{MRI}$ in diagnosing preoperative staging, assessing treatment response, predicting prognosis and histopathological features of gastric cancer, and determining the most appropriate treatment plan. With the improvements of scan technique, functional MRI, and MRI-based radiomics analysis, more and more experimental studies and clinical trials will be conducted, further affirming the advancement and reliability of MRI in gastric cancer.

\section{Conflict of interest disclosure}

The authors declared no conflicts of interest.

\section{References}

1. Bray F, Ferlay J, Soerjomataram I, Siegel RL, Torre LA, Jemal A. Global cancer statistics 2018: GLOBOCAN estimates of incidence and mortality worldwide for 36 cancers in 185 countries. CA Cancer J Clin 2018; 68:394-424. [Crossref]
2. Chen WQ, Zheng RS, Zhang SW, et al. Cancer incidence and mortality in China, 2013. Cancer Lett 2017; 401:63-71. [Crossref]

3. Allemani C, Matsuda T, Di Carlo V, et al. Global surveillance of trends in cancer survival 200014 (CONCORD-3): analysis of individual records for 37513025 patients diagnosed with one of 18 cancers from 322 population-based registries in 71 countries. Lancet 2018; 391:10231075. [Crossref]

4. Ly QP, Sasson AR. Modern surgical considerations for gastric cancer. J Natl Compr Canc Netw 2008; 6:885-894. [Crossref]

5. Motohara T, Semelka RC. MRI in staging of gastric cancer. Abdom Imaging 2002; 27:376-383. [Crossref]

6. Qayyum A. Diffusion-weighted imaging in the abdomen and pelvis: concepts and applications. Radiographics 2009; 29:1797-1810. [Crossref]

7. Bonekamp S, Corona-Villalobos CP, Kamel IR. Oncologic applications of diffusion-weighted MRI in the body. J Magn Reson Imaging 2012; 35:257-279. [Crossref]

8. Koh DM, Collins DJ. Diffusion-weighted MRI in the body: applications and challenges in oncology. AJR Am J Roentgenol 2007; 188:1622-1635. [Crossref]

9. $\mathrm{Li} H \mathrm{HH}, \mathrm{Zhu} H$, Yue $\mathrm{L}$, et al. Feasibility of free-breathing dynamic contrast-enhanced MRI of gastric cancer using a golden-angle radial stack-of-stars VIBE sequence: comparison with the conventional contrast-enhanced breath-hold 3D VIBE sequence. Eur Radiol 2018; 28:1891-1899. [Crossref]

10. Cho EY, Kim SH, Yoon JH, et al. Apparent diffusion coefficient for discriminating metastatic from non-metastatic lymph nodes in primary rectal cancer. Eur J Radiol 2013; 82:e662-668. [Crossref]

11. In $\mathrm{H}$, Ravetch $\mathrm{E}$, Langdon-Embry $\mathrm{M}$, et al. The newly proposed clinical and post-neoadjuvant treatment staging classifications for gastric adenocarcinoma for the American Joint Committee on Cancer (AJCC) staging. Gastric Cancer 2018; 21:1-9. [Crossref]

12. Kwee RM, Kwee TC. Imaging in local staging of gastric cancer: a systematic review. J Clin Oncol 2007; 25:2107-2116. [Crossref]

13. Botet JF, Lightdale CJ, Zauber AG, et al. Preoperative staging of gastric cancer: comparison of endoscopic US and dynamic CT. Radiology 1991; 181:426-432. [Crossref]

14. Habermann $C R$, Weiss $F$, Riecken $R$, et al. Preoperative staging of gastric adenocarcinoma: comparison of helical CT and endoscopic US. Radiology 2004; 230:465-471. [Crossref]

15. Hwang SW, Lee $\mathrm{DH}$, Lee $\mathrm{SH}$, et al. Preoperative staging of gastric cancer by endoscopic ultrasonography and multidetector-row computed tomography. J Gastroenterol Hepatol 2010; 25:512-518. [Crossref]

16. Kim AY, Kim HJ, Ha HK. Gastric cancer by multidetector row CT: preoperative staging. Abdom Imaging 2005; 30:465-472. [Crossref]

17. Makino T, Fujiwara $\mathrm{Y}$, Takiguchi $\mathrm{S}$, et al. Preoperative $T$ staging of gastric cancer by multi-detector row computed tomography. Surgery 2011; 149:672-679. [Crossref]

18. Kim Jl, Kim YH, Lee KH, et al. Type-specific diagnosis and evaluation of longitudinal tumor extent of borrmann type IV gastric cancer: CT versus gastroscopy. Korean J Radiol 2013; 14:597-606. [Crossref]
19. Polkowski M, Palucki J, Wronska E, Szawlowski A, Nasierowska-Guttmejer A, Butruk E. Endosonography versus helical computed tomography for locoregional staging of gastric cancer. Endoscopy 2004; 36:617-623. [Crossref]

20. Yang DM, Kim HC, Jin W, et al. 64 multidetector-row computed tomography for preoperative evaluation of gastric cancer: histological correlation. J Comput Assist Tomogr 2007; 31:98-103. [Crossref]

21. Ahn HS, Lee HJ, Yoo MW, et al. Diagnostic accuracy of $\mathrm{T}$ and $\mathrm{N}$ stages with endoscopy, stomach protocol $\mathrm{CT}$, and endoscopic ultrasonography in early gastric cancer. J Surg Oncol 2009; 99:20-27. [Crossref]

22. Kwee RM, Kwee TC. Modern imaging techniques for preoperative detection of distant metastases in gastric cancer. World J Gastroenterol 2015; 21:10502-10509. [Crossref]

23. Kang BC, Kim JH, Kim KW, et al. Value of the dynamic and delayed MR sequence with Gd-DTPA in the T-staging of stomach cancer: correlation with the histopathology. Abdom Imaging 2000; 25:14-24. [Crossref]

24. Sohn KM, Lee JM, Lee SY, Ahn BY, Park SM, Kim $\mathrm{KM}$. Comparing MR imaging and $\mathrm{CT}$ in the staging of gastric carcinoma. AJR Am J Roentgenol 2000; 174:1551-1557. [Crossref]

25. Wang CK, Kuo YT, Liu GC, Tsai KB, Huang YS. Dynamic contrast-enhanced subtraction and delayed MRI of gastric tumors: radiologic-pathologic correlation. J Comput Assist Tomogr 2000; 24:872-877. [Crossref]

26. Arslan H, Fatih Ozbay M, Calli I, et al. Contribution of diffusion weighted MRI to diagnosis and staging in gastric tumors and comparison with multi-detector computed tomography. Radiol Oncol 2017; 51:23-29. [Crossref]

27. Liu S, He J, Guan W, et al. Added value of diffusion-weighted MR imaging to T2-weighted and dynamic contrast-enhanced MR imaging in T staging of gastric cancer. Clin Imaging 2014; 38:122-128. [Crossref]

28. Caivano R, Rabasco P, Lotumolo A, et al. Gastric cancer: The role of diffusion weighted imaging in the preoperative staging. Cancer Invest 2014; 32:184-190. [Crossref]

29. Jang KM, Kim SH, Lee SJ, Lee MW, Choi D, Kim KM. Upper abdominal gadoxetic acid-enhanced and diffusion-weighted MRI for the detection of gastric cancer: Comparison with two-dimensional multidetector row CT. Clin Radiol 2014; 69:827-835. [Crossref]

30. Joo I, Lee JM, Kim JH, Shin Cl, Han JK, Choi BI. Prospective comparison of $3 \mathrm{~T}$ MRI with diffusion-weighted imaging and MDCT for the preoperative TNM staging of gastric cancer. J Magn Reson Imaging 2015; 41:814-821. [Crossref]

31. Monig SP, Zirbes TK, Schroder W, et al. Staging of gastric cancer: correlation of lymph node size and metastatic infiltration. AJR Am J Roentgenol 1999; 173:365-367. [Crossref]

32. Park HS, Kim YJ, Ko SY, et al. Benign regional lymph nodes in gastric cancer on multidetector row CT. Acta Radiol 2012; 53:501-507. [Crossref]

33. Tatsumi $\mathrm{Y}$, Tanigawa N, Nishimura H, et al. Preoperative diagnosis of lymph node metastases in gastric cancer by magnetic resonance imaging with ferumoxtran-10. Gastric Cancer 2006; 9:120-128. [Crossref] 
34. Giganti F, Ambrosi A, Chiari D, et al. Apparent diffusion coefficient by diffusion-weighted magnetic resonance imaging as a sole biomarker for staging and prognosis of gastric cancer. Chin J Cancer Res 2017; 29:118-126. [Crossref]

35. Liu S, Wang H, Guan W, et al. Preoperative apparent diffusion coefficient value of gastric cancer by diffusion-weighted imaging: Correlations with postoperative TNM staging. J Magn Reson Imaging 2015; 42:837-843. [Crossref]

36. Cheng J, Wang Y, Deng J, et al. Discrimination of metastatic lymph nodes in patients with gastric carcinoma using diffusion-weighted imaging. J Magn Reson Imaging 2013; 37:1436-1444. [Crossref]

37. Shinya S, Sasaki T, Nakagawa Y, Guiquing Z, Yamamoto $F$, Yamashita $Y$. The usefulness of diffusion-weighted imaging (DWI) for the detection of gastric cancer. Hepatogastroenterology 2007; 54:1378-1381.

38. Zhong J, Zhao W, Ren F, et al. Lymph node metastasis in patients with gastric cancer: a multi-modality, morphologic and functional imaging study. Am J Transl Res 2016; 8:56015609.

39. Hasbahceci M, Akcakaya A, Memmi N, et al. Diffusion MRI on lymph node staging of gastric adenocarcinoma. Quant Imaging Med Surg 2015; 5:392-400.

40. Soussan M, Des Guetz G, Barrau V, et al. Comparison of FDG-PET/CT and MR with diffusion-weighted imaging for assessing peritoneal carcinomatosis from gastrointestinal malignancy. Eur Radiol 2012; 22:1479-1487. [Crossref]

41. Lee DH, Kim SH, Joo I, Hur BY, Han JK. Comparison between 18F-FDG PET/MRI and MDCT for the assessment of preoperative staging and resectability of gastric cancer. Eur J Radiol 2016; 85:1085-1091. [Crossref]

42. Liu Y, Zheng D, Liu JJ, et al. Comparing PET/ MRI with PET/CT for Pretreatment Staging of Gastric Cancer. Gastroenterol Res Pract 2019; 2019:9564627. [Crossref]

43. Cunningham $D$, Allum $W H$, Stenning $S P$, et al. Perioperative chemotherapy versus surgery alone for resectable gastroesophageal cancer. N Engl J Med 2006; 355:11-20. [Crossref]

44. Ychou M, Boige V, Pignon JP, et al. Perioperative chemotherapy compared with surgery alone for resectable gastroesophageal adenocarcinoma: an FNCLCC and FFCD multicenter phase III trial. J Clin Oncol 2011; 29:1715-1721. [Crossref]

45. Rowley HA, Grant PE, Roberts TP. Diffusion MR imaging. Theory and applications. Neuroimaging Clin N Am 1999; 9:343-361.

46. Padhani AR, Liu G, Koh DM, et al. Diffusion-weighted magnetic resonance imaging as a cancer biomarker: consensus and recommendations. Neoplasia 2009; 11:102-125. [Crossref]

47. Yankeelov TE, Lepage M, Chakravarthy A, et al. Integration of quantitative DCE-MRI and ADC mapping to monitor treatment response in human breast cancer: initial results. Magn Reson Imaging 2007; 25:1-13. [Crossref]
48. De Cobelli F, Giganti F, Orsenigo E, et al. Apparent diffusion coefficient modifications in assessing gastro-oesophageal cancer response to neoadjuvant treatment: comparison with tumour regression grade at histology. Eur Radiol 2013; 23:2165-2174. [Crossref]

49. Giganti F, De Cobelli F, Canevari C, et al. Response to chemotherapy in gastric adenocarcinoma with diffusion-weighted MRI and (18) F-FDG-PET/CT: correlation of apparent diffusion coefficient and partial volume corrected standardized uptake value with histological tumor regression grade. J Magn Reson Imaging 2014; 40:1147-1157. [Crossref]

50. Zhong J, Zhao W, Ma W, et al. DWl as a quantitative biomarker in predicting chemotherapeutic efficacy at multitime points on gastric cancer lymph nodes metastases. Medicine (Baltimore) 2016; 95:e3236. [Crossref]

51. Lee DH, Kim SH, Im SA, Oh DY, Kim TY, Han JK. Multiparametric fully-integrated 18-FDG PET/ MRI of advanced gastric cancer for prediction of chemotherapy response: a preliminary study. Eur Radiol 2016; 26:2771-2778. [Crossref]

52. Cheng J, Wang Y, Zhang CF, et al. Chemotherapy response evaluation in a mouse model of gastric cancer using intravoxel incoherent motion diffusion-weighted MRI and histopathology. World J Gastroenterol 2017; 23:1990-2001. [Crossref]

53. Song XL, Kang HK, Jeong GW, et al. Intravoxel incoherent motion diffusion-weighted imaging for monitoring chemotherapeutic efficacy in gastric cancer. World J Gastroenterol 2016; 22:5520-5531. [Crossref]

54. Liu S, Guan W, Wang H, et al. Apparent diffusion coefficient value of gastric cancer by diffusion-weighted imaging: correlations with the histological differentiation and Lauren classification. Eur J Radiol 2014; 83:2122-2128. [Crossref]

55. Liu S, Zheng $\mathrm{H}$, Zhang $\mathrm{Y}$, et al. Whole-volume apparent diffusion coefficient-based entropy parameters for assessment of gastric cancer aggressiveness. J Magn Reson Imaging 2018; 47:168-175. [Crossref]

56. Zhang Y, Chen J, Liu S, et al. Assessment of histological differentiation in gastric cancers using whole-volume histogram analysis of apparent diffusion coefficient maps. J Magn Reson Imaging 2017; 45:440-449. [Crossref]

57. Ma L, Xu X, Zhang M, et al. Dynamic contrast-enhanced MRI of gastric cancer: Correlations of the pharmacokinetic parameters with histological type, Lauren classification, and angiogenesis. Magn Reson Imaging 2017; 37:27-32. [Crossref]

58. Ji C, Zhang Q, Guan W, et al. Role of intravoxe incoherent motion MR imaging in preoperative assessing HER2 status of gastric cancers. Oncotarget 2017; 8:49293-49302. [Crossref]

59. Ji C, Chen L, Guan W, et al. Intravoxel incoherent motion magnetic resonance imaging in assessing histopathological features of gastric cancers: initial findings. Transl Cancer Res 2017; 6:1129-1140. [Crossref]
60. Ito A, Ito $Y$, Matsushima S, et al. New whole-body multimodality imaging of gastric cancer peritoneal metastasis combining fluorescence imaging with ICG-labeled antibody and MRI in mice. Gastric Cancer 2014; 17:497-507. [Crossref]

61. Yan X, Song X, Wang Z. Construction of specific magnetic resonance imaging/optical dual-modality molecular probe used for imaging angiogenesis of gastric cancer. Artif Cells Nanomed Biotechnol 2017; 45:399-403. [Crossref]

62. Chen J, Ren G, Cai R, et al. Cellular magnetic resonance imaging: in vivo tracking of gastric cancer cells and detecting of lymph node metastases using microparticles of iron oxide in mice. Cancer Manag Res 2019; 11:7317-7326. [Crossref]

63. Ding N, Sano K, Kanazaki K, et al. In Vivo HER2-Targeted Magnetic Resonance Tumor Imaging Using Iron Oxide Nanoparticles Conjugated with Anti-HER2 Fragment Antibody. Mol Imaging Biol 2016; 18:870-876. [Crossref]

64. Chen Y, Wang W, Lian G, et al. Development of an MRI-visible nonviral vector for siRNA delivery targeting gastric cancer. Int J Nanomedicine 2012; 7:359-368. [Crossref]

65. Chen Y, Lian G, Liao C, et al. Characterization of polyethylene glycol-grafted polyethylenimine and superparamagnetic iron oxide nanoparticles (PEG-g-PEI-SPION) as an MRI-visible vector for siRNA delivery in gastric cancer in vitro and in vivo. J Gastroenterol 2013; 48:809-821. [Crossref]

66. Corradini S, Alongi $\mathrm{F}$, Andratschke $\mathrm{N}$, et al. MR-guidance in clinical reality: current treatment challenges and future perspectives. Radiat Oncol 2019; 14:92. [Crossref]

67. Pollard JM, Wen Z, Sadagopan R, Wang J, Ibbott GS. The future of image-guided radiotherapy will be MR guided. Br J Radiol 2017; 90:20160667. [Crossref]

68. Chun SJ, Jeon SH, Chie EK. A case report of salvage radiotherapy for a patient with recurrent gastric cancer and multiple comorbidities using real-time MRI-guided adaptive treatment system. Cureus 2018; 10:e2471. [Crossref]

69. Tanaka O, Omatsu T, Kariya S, et al. Usefulness of diffusion-weighted magnetic resonance imaging for evaluating the effect of hemostatic radiotherapy for unresectable gastric cancer. Clin J Gastroenterol 2019; 12:269-273. [Crossref]

70. Wang S, Kim S, Chawla S, et al. Differentiation between glioblastomas, solitary brain metastases, and primary cerebral lymphomas using diffusion tensor and dynamic susceptibility contrast-enhanced MR imaging. AJNR Am J Neuroradiol 2011; 32:507-514. [Crossref]

71. Nakamura K, Joja I, Nagasaka T, et al. The mean apparent diffusion coefficient value (ADCmean) on primary cervical cancer is a predictive marker for disease recurrence. Gynecol Oncol 2012; 127:478-483. [Crossref]

72. Giganti F, Orsenigo E, Esposito A, et al. Prognostic role of diffusion-weighted MR imaging for resectable gastric cancer. Radiology 2015; 276:444-452. [Crossref] 